\title{
Symbolic Generation of Finite Difference Formulas*
}

\author{
By H. B. Keller and V. Pereyra
}

\begin{abstract}
Tables of coefficients for high order accurate, compact approximations to the first ten derivatives on and at the midpoints of uniform nets are presented. The exact rational weights are generated and tested by means of symbolic manipulation implemented through MACSYMA. These weights are required in the application of deferred corrections to new methods for solving higher order two point boundary value problems.
\end{abstract}

1. Introduction. Compact difference schemes are, by definition, those which use the least number of net points to obtain consistent approximations (i.e. at least first order accurate). Extending this definition, higher order compact schemes are those which use the least number of net points to obtain higher order accurate approximations. In this paper we derive and present tables of the coefficients for higher order compact approximations, from accuracy $h^{\mathbf{2}}$ to $h^{\mathbf{1 0}}$, to the first 10 derivatives of smooth functions on uniform nets. These approximations are particularly useful in applications of deferred corrections, and this was part of the motivation for the present work [5]. Fast weight generators have also. been used for this purpose [1] , [2] but the current tables are more efficient. In addition, our tables are motivated by new schemes for solving higher order O.D.E. boundary value problems [5]

The derivation of the formulas is classical, involving no more than Taylor expansions. However, the work is tedious and very prone to errors. Thus, we have used symbolic manipulation, implemented through MACSYMA, [6], both to derive the coefficients and to independently check their correctness. The coefficients are rational numbers and are given in their exact form as quotients of integers. Partial tables of some such coefficients have been published in [1], [7], [8], [9]. The suggestion to use MACSYMA was made by the Numerical Analysis Group at Stanford University. The second author would like to thank Professor Gene H. Golub for his hospitality while visiting Stanford during the summer of 1975 , where part of this work was carried out.

2. Preliminaries. Given a uniform net $\left\{t_{j}\right\}$ with step size $h$ and a smooth function $f(t)$ we study compact approximations to the derivatives of $f(t)$ at $t=0$ and at $t=h / 2$, using only function values $\left\{f\left(t_{i}\right)\right\}$. We prefer compact approximations since they have truncation error expansions in powers of $h^{2}$, they use a minimum number of ordinates for a given order of accuracy and have the smallest weights and error constants. We use the notation $D^{p} f(\theta) \equiv d^{p} f(\theta) / d t^{p}, \theta=0$ or $h / 2$, and $D_{2 q}^{p, \theta} f$ for the

Received September 12, 1977.

AMS (MOS) subject classifications (1970). Primary 65D25; Secondary 65L05, 65L10, 68A1 5.

* This work was supported under Contract No. AT-04-3-767, Project Agreement 12 with the Energy Research and Development Administration. 
compact approximation of order $2 q$ to $D^{p} f(\theta)$. These approximations are of the form

$$
h^{p} D_{2 q}^{p, \theta} f \equiv \sum_{j=-\underline{m} p, q}^{\bar{m}_{p, q}} w_{j, \theta}^{p, q} f\left(t_{j}\right)=h^{p} D^{p} f(\theta)+o\left(h^{p+2 q}\right) .
$$

The index values $\underline{m}_{p, q}, \bar{m}_{p, q}$, and the number of mesh points involved $n_{p, q}=\underline{m}_{p, q}$ $+\bar{m}_{p, q}+1$ for the various possible cases are given in Table $\mathrm{A}$.

TABLE A

Index values and number of net points for the compact difference approximations $D_{2 q}^{p, \theta} f$

\begin{tabular}{|c|c|l|l|}
\cline { 3 - 4 } \multicolumn{2}{c|}{} & $\theta=0$ & $\theta=h / 2$ \\
\hline \hline \multirow{3}{*}{$p$ even } & $m_{p, q}$ & $p / 2+q-1$ & $p / 2+q-1$ \\
\hline \multirow{2}{*}{$p$ odd } & $n_{p, q}$ & $p+2 q-1$ & $p+2 q$ \\
\hline \hline & $m_{p, q}$ & $(p+1) / 2+q-1$ & $(p-1) / 2+q-1$ \\
\cline { 2 - 4 } & $n_{p, q}$ & $p+2 q$ & $p+2 q-1$ \\
\hline \hline & $\bar{m}_{p, q}$ & $m_{p, q}$ & $m_{p, q}+1$ \\
\hline
\end{tabular}

We shall describe a general method for calculating the weights $w_{s, \theta}^{p, q}$ and give tables of them for the range $1 \leqslant p \leqslant 10,1 \leqslant q \leqslant 5$. The method is based on wellknown, even classical, facts that we now recall [3], [4]. The basic finite difference operators that we use are:

$$
\begin{aligned}
D_{+} v_{j} & \equiv h^{-1}\left(v_{j+1}-v_{j}\right), & & D_{-} v_{j} \equiv h^{-1}\left(v_{j}-v_{j-1}\right), \\
D_{0} v_{j} & \equiv(2 h)^{-1}\left(v_{j+1}-v_{j-1}\right), & & M_{ \pm} v_{j} \equiv 2^{-1}\left(v_{j \pm 1}+v_{j}\right), \\
D_{+} D_{-} v_{j} & =h^{-2}\left(v_{j+1}-2 v_{j}+v_{j-1}\right) . & &
\end{aligned}
$$

Each of these operators has a formal asymptotic expansion in powers of $h^{2}$. For smooth $v(t)$ we obtain by Taylor expansions about the appropriate abscissa, $v_{j}$ or $v_{j \pm 1 / 2}$ :

$$
\begin{aligned}
& D_{ \pm} v_{j} \equiv\left[D+\sum_{\nu=1}^{L} \frac{D^{2 \nu+1}}{4^{\nu}(2 \nu+1) !} h^{2 \nu}\right] v_{j \pm 1 / 2}+O\left(h^{2 L+2}\right), \\
& M_{ \pm} v_{j} \equiv\left[I+\sum_{\nu=1}^{L} \frac{D^{2 \nu}}{4^{\nu}(2 \nu) !} h^{2 \nu}\right] v_{j \pm 1 / 2}+O\left(h^{2 L+2}\right), \\
& D_{0} v_{j} \equiv\left[D+\sum_{\nu=1}^{L} \frac{D^{2 \nu+1}}{(2 \nu+1) !} h^{2 \nu}\right] v_{j}+O\left(h^{2 L+2}\right),
\end{aligned}
$$




$$
D_{+} D_{-} v_{j}=\left[D^{2}+\sum_{\nu=1}^{L} 2 \frac{D^{2 \nu+2}}{(2 v+2) !} h^{2 \nu}\right] v_{j}+O\left(h^{2 L+2}\right) .
$$

Here $v_{j \pm 1 / 2}$ stands for $v\left(t_{j} \pm 1 / 2 h\right)$, and $I$ is the identity operator. Clearly, these finite difference formulas are all $O\left(h^{2}\right)$ approximations to the first term in each right hand side.

Approximations of order $h^{2}$ to higher order derivatives at $t_{j}-\theta$ can now be easily obtained by appropriate combinations of these basic operators. We denote by index " $e$ " (even) those approximations for $\theta=0$, and by index " $\phi$ " (odd) those for $\theta=h / 2$. The approximations are defined as

$$
\begin{aligned}
D_{2}^{2 \mu, e} v_{j} & \equiv\left(D_{+} D_{-}\right)^{\mu} v_{j}, \\
D_{2}^{2 \mu, \phi} v_{j} & \equiv\left(D_{+} D_{-}\right)^{\mu} M_{-} v_{j}, \\
D_{2}^{2 \mu+1, e} v_{j} & \equiv\left(D_{+} D_{-}\right)^{\mu} D_{0} v_{j}, \\
D_{2}^{2 \mu+1, \phi^{2}} v_{j} & \equiv\left(D_{+} D_{-}\right)^{\mu} D_{-} v_{j} .
\end{aligned}
$$

A little reflection shows that formal asymptotic expansions for these higher order operators can be obtained by simply using the expansions (2.3). Thus, for example, using (2.3d) in (2.4a) yields:

$$
D_{2}^{2 \mu, e} v_{j} \equiv\left[D^{2}+\sum_{\nu=1}^{L} h^{2 \nu} 2 \frac{D^{2 \nu+2}}{(2 \nu+2) !}\right]^{\mu} v_{j}+O\left(h^{2 L+2}\right) .
$$

Clearly, this has the expanded form, with rational coefficients, $C_{2, \nu}^{2 \mu, e}$ :

$$
D_{2}^{2 \mu, e} v_{j} \equiv\left[D^{2 \mu}+\sum_{\nu=1}^{L} h^{2 \nu} C_{2 \nu}^{2 \mu, e} D^{2(\mu+\nu)}\right] v_{j}+O\left(h^{2 L+2}\right) .
$$

Similar expansions exist for the other formulas in (2.4) and so they are all $O\left(h^{2}\right)$ approximations to the corresponding derivatives. To explicitly determine the coefficients $C_{2, \nu}^{2 \mu, e}$ we need only formally expand $[\cdots]^{\mu}$ in (2.5) into powers of $h^{2}$. This is one of the tedious and error prone computations that we do symbolically.

Our method for constructing higher order approximations is contained in the next theorem.

THEOREM 2.7. Centered difference approximations accurate to order $2 k$ for a derivative of order $p$ can be obtained recursively as:

$$
D_{2 k}^{p, \theta} \equiv D_{2(k-1)}^{p, \theta}-h^{2(k-1)} C_{2(k-1), k-1}^{p, \theta} D_{2}^{p+2(k-1), \theta}, \quad k=2, \ldots
$$

The operators $D_{2 k}^{p, \theta}$ so defined have the asymptotic expansions:

$$
D_{2 k}^{p, \theta}=D^{p}+\sum_{\nu=k}^{L} h^{2 \nu} C_{2 k, \nu}^{p, \theta} D^{p+2 \nu}+O\left(h^{2 L+2}\right) .
$$

Proof. The proof is by induction on $k$. The expansions for $k=1$ are obtained 
as in (2.5)-(2.6) by using (2.3) in (2.4). Assume that for $k \geqslant 2$, any $p \geqslant 0, \theta=e$ or $\phi$ we have

$$
D_{2(k-1)}^{p, \theta}=D^{p}+\sum_{\nu=k-1}^{L} h^{2 \nu} C_{2(k-1), \nu}^{p, \theta} D^{p+2 \nu}+O\left(h^{2 L+2}\right) .
$$

Using these expansions in (2.8), we obtain

$$
\begin{aligned}
D_{2 k}^{p, \theta} \equiv & D^{p}+\sum_{\nu=k-1}^{L} h^{2 \nu} C_{2(k-1), \nu}^{p, \theta} D^{p+2 \nu} \\
& -h^{2(k-1)} C_{2(k-1), k-1}^{p, \theta}\left[D^{p+2(k-1)}+\sum_{\nu=1}^{L} h^{2 \nu} C_{2, \nu}^{p+2(k-1), \theta} D^{p+2(k+\nu-1)}\right] \\
& +O\left(h^{2 L+1}\right) .
\end{aligned}
$$

This simplifies, after collecting terms in like powers of $h$, to the form (2.9). This completes the induction argument, proves the theorem, and yields the recursive formula for the coefficients:

$$
C_{2 k, \nu}^{p, \theta}=C_{2(k-1), \nu}^{p, \theta}-C_{2(k-1), k-1}^{p, \theta} C_{2, \nu-(k-1), \theta}^{p+2(k-1),}, \quad \nu \geqslant k .
$$

3. Construction of the Finite Difference Operators. Although Theorem 2.7 gives us a recipe for constructing high order approximations to any derivative, it does not actually provide the formulas (2.1). In order to obtain the weights $w_{s, \theta}^{p, q}$ we use the shift operator $E v_{j} \equiv v_{j+1}$ in (2.1) which can then be written as:

$$
h^{p} D_{2 q}^{p, \theta} f=\sum_{j=-\underline{m}_{p, q}}^{\bar{m}_{p, q}} w_{j, \theta}^{p, q} E^{j} f(\theta) .
$$

Also, the basic operators in (2.2) become

$$
\begin{aligned}
h D_{+} & \equiv(E-I), \\
h D_{-} & \equiv\left(I-E^{-1}\right), \\
h D_{0} & \equiv 2^{-1}\left(E-E^{-1}\right), \\
M_{+} & \equiv 2^{-1}(E+I), \\
M_{-} & \equiv 2^{-1}\left(E^{-1}+I\right), \\
h^{2} D_{+} D_{-} & \equiv\left(E-2 I+E^{-1}\right) .
\end{aligned}
$$

Since $E^{j}$ is linear and it commutes with $E^{i}$ for any integers $i, j$, we can operate symbolically with these expressions as if they were rational functions in the variable $E$. Then, by (2.4) and (2.8) we can construct recursively the operators $h^{p} D_{2 q}^{p, \theta}$ and obtain the actual weights $w_{s, \theta}^{p, q}$.

We first observe that in

$$
h^{p} D_{2 k}^{p, \theta}=h^{p} D_{2(k-1)}^{p, \theta}-C_{2(k-1), k-1}^{p, \theta}\left(D_{2}^{p+2(k-1), \theta} h^{p+2(k-1)}\right)
$$


each difference operator is multiplied by an appropriate power of $h$ so that no $h$ 's will appear in these formulas. Specifically, if we introduce

$$
\begin{aligned}
\widetilde{D}_{2}^{2 \mu, e} & \equiv h^{2 \mu} D_{2}^{2 \mu, e} \equiv\left(E-2 I+E^{-1}\right)^{\mu}, \\
\widetilde{D}_{2}^{2 \mu, \phi} & \equiv h^{2 \mu} D_{2}^{2 \mu, \phi} \equiv 1 / 2\left(E-2 I+E^{-1}\right)^{\mu}\left(E^{-1}+I\right), \\
\widetilde{D}_{2}^{2 \mu+1, e} & \equiv h^{2 \mu+1} D_{2}^{2 \mu+1, e} \equiv 1 / 2\left(E-2 I+E^{-1}\right)^{\mu}\left(E-E^{-1}\right), \\
\widetilde{D}_{2}^{2 \mu+1, \phi} & \equiv h^{2 \mu+1} D_{2}^{2 \mu+1, \phi} \equiv\left(E-2 I+E^{-1}\right)^{\mu}\left(I-E^{-1}\right),
\end{aligned}
$$

then the recursion (3.3) becomes:

$$
\begin{aligned}
\widetilde{D}_{2 k}^{p, \theta} \equiv \widetilde{D}_{2(k-1)}^{p, \theta}-C_{2(k-1), k-1}^{p, \theta} \widetilde{D}_{2}^{p+2(k-1), \theta}, \\
\quad k=2,3, \ldots ; q=0,1, \ldots ; \theta=0, h / 2 .
\end{aligned}
$$

Now by rearranging $\widetilde{D}_{2 k}^{p, \theta}$ as a linear combination of positive and negative powers of $E$ we obtain (3.1). That is the coefficient of $E^{S}$ in this linear combination is the weight $w_{s, \theta}^{p, k}$.

4. Determination of Weights by Symbolic Manipulation. The entire procedure to construct the weights $w_{s, \theta}^{p, q}$ consists then of the following steps:

(A) Construct expansions for $D_{2}^{p, \theta}$ from those given in (2.3).

(B) Construct expansions for $D_{2 k}^{p, \theta}$ using the recursion (2.8) and (A). This provides the coefficients $C_{2 k, k}^{p, \theta}$.

(C) Construct $\widetilde{D}_{2}^{p, \theta}$ by means of (3.4).

(D) Obtain $\widetilde{D}_{2 k}^{p, \theta}$ recursively by using (3.5) and the coefficients $C_{2(k-1),(k-1)}^{p, \theta}$ from (B).

These tasks involve only multiplication of asymptotic expansions (for (A) and (B)) and multiplication of polynomials in positive and negative powers of $E$. All the numerical coefficients involved are rational. It is a very tedious task to compile fairly extensive tables covering the range needed in most applications, even with the aid of a computer. This is where MACSYMA comes to our rescue.

MACSYMA (Project MAC's SYmbolic MAnipulation system) [6] is a large computer programming system written in LISP and used for performing symbolic as well as numerical mathematical manipulations. This sytem is available on a DEC PDP. 10 Computer at MIT and can be accessed through the ARPA Network. MACSYMA has facilities for the symbolic manipulation of truncated Taylor series, operations with rational functions, and indefinite precision computations with rational numbers, all of which are very convenient for our purposes.

As an example of the main tasks performed we show in the Appendix an actual MACSYMA dialogue to obtain the weights in $\widetilde{D}_{6}^{2, e}$. Of course, we have used many other powerful facilities of MACSYMA in order to produce efficiently the wealth of formulas required. What is more important perhaps, is that we have used MACSYMA to make an independent check on the final formulas obtained and to output the tables of the Appendix. Thus, so far as we can determine, the coefficients given in Tables 1-10 are the exact rational weights in the corresponding formulas. 
The independent check consists in applying symbolically the computed formulas $\widetilde{D}_{2 k}^{q, \theta}$ to various powers of $x$ and $(x+h / 2)$ and comparing them with the exact derivatives, $h^{q} D^{q}$, of those powers. In particular,

$$
\widetilde{D}_{2 k}^{q, \theta} x^{p}-h^{q} D^{q}(x+\theta)^{p}
$$

should be zero for $p<q+2 k$ and should be a known multiple of $h^{2 k}$ for $p=q+2 k$.

We have checked this for all the formulas presented (see (C34) in Appendix).

We have printed only half the number of weights taking advantage of the various symmetries and antisymmetries listed below:

$$
\begin{aligned}
& \text { (a) } w_{j, e}^{2 r, q}=w_{-j, e}^{2 r, q}, \\
& \text { (b) } w_{j, e}^{2 r+1, q}=-w_{-j, e}^{2 r+1, q}, \\
& \text { (c) } w_{j, \phi}^{2 r, q}=w_{-j+1, \phi}^{2 r, q}, \\
& \text { (d) } w_{j, \phi}^{2 r+1, q}=-w_{-j+1, \phi}^{2 r+1, q} .
\end{aligned}
$$

The weights are given in Tables $1-10$ for $j \geqslant 0$.

For completeness we include also in Tables 11 and 12 the coefficients $C_{2 k, k}^{p, \theta}$ used in the construction of the $w$ 's.

Appendix. In this Appendix we present a self-contained example of the use of MACSYMA to obtain the weights in $\widetilde{D}_{6}^{2, e}$, following the procedure of Section 4 .

In order to make the following MACSYMA dialogue comprehensible to the uninitiated we will give now the minimum of explanation necessary to understand it. We assume a knowledge of some high level language, like FORTRAN or ALGOL. Lines labeled (C - - ) are input by the user, while those labelled (D - -) or (E - -) are the computer answers.

(C26) assigns to $D H[2,2]$ the value $\Sigma_{i=1}^{7} 2 D^{2 i} H^{2(i-1)} /(2 i)$ !. This represents the first 7 terms of the expansion of $D_{2}^{2, e}$, as given in (2.3d). (D26) is then the display of this sum.

In general, $D H[n, m]$ will contain the first few terms of the asymptotic expansion of $D_{m}^{n, e}$. In (C27) we obtain $D_{2}^{4, e}$ by using the TAYLOR command that produces the truncated Taylor expansion of $(D H[2,2])^{2}$ in the variable $D$ up to terms in $D^{14}$.

Then $D_{4}^{2, e}$, the fourth order approximation to $D^{2}$, is obtained by subtracting from $D_{2}^{2, e}$ an $O\left(h^{2}\right)$ approximation to $h^{2} D^{4} / 12$, namely $D H[4,2] * H * * 2 / 12$.

With this expansion we have enough information to generate $\widetilde{D}_{6}^{2, e}$, which is done in (C29)-(C33). Here again we store $\widetilde{D}_{m}^{n, e}$ in $E H[n, m]$. The only new feature is the command RATEXPAND which simply expands the operand by multiplying out exponentiated sums, etc., in order to give the resulting formulas displayed in (D29)-(D33). The desired weights are the coefficients in (D33).

Finally, our function $\mathrm{F} 6$ performs on $E H[2,6] \equiv \widetilde{D}_{6}^{2, e}$ the check indicated at the end of Section 4 , where $p=8$. For completeness we display the function F6 and the auxiliary functions F2, F3 and F4, although we shall not explain them here. 


\section{Printout}

(C26) $D H[2,2]: \operatorname{sum}(2 * D * *(2 * I) * H * *(2 *(I-1)) /(2 * I) !, I, 1,7)$;

(D26) $\frac{D^{14} H^{12}}{43589145600}+\frac{D^{12} H^{10}}{239500800}+\frac{D^{10} H^{8}}{1814400}+\frac{D^{8} H^{6}}{20160}+\frac{D^{6} H^{4}}{360}$

$$
+\frac{D^{4} H^{2}}{12}+D^{2}
$$

(C27) $D H[4,2]: \operatorname{TAYLOR}(D H[2,2] * * 2, D, 0,14)$;

(D27) $\mid R / D^{4}+\frac{H^{2} D^{6}}{6}+\frac{H^{4} D^{8}}{80}+\frac{17 H^{6} D^{10}}{30240}+\frac{31 H^{8} D^{12}}{1814400}+\frac{H^{10} D^{14}}{2661120}+\cdots$

(C28) $D H[2,4]: \operatorname{TAYLOR}(D H[2,2]-D H[4,2] * H * * 2 / 12, D, 0,12)$;

(D28) $\mid R / D^{2}-\frac{H^{4} D^{6}}{90}-\frac{H^{6} D^{8}}{1008}-\frac{H^{8} D^{10}}{21600}-\frac{17 H^{10} D^{12}}{11975040}+\cdots$

(C29) $E H[2,2]: E-2+E * *(-1)$;

(D29) $E+\frac{1}{E}-2$

(C31) $E H[4,2]: \operatorname{RATEXPAND}(E H[2,2] * * 2)$;

(D31) $E^{2}-4 E-\frac{4}{E}+\frac{1}{E^{2}}+6$

(C32) $E H[2,4]: \operatorname{RATEXPAND}(E H[2,2]-E H[4,2] / 12)$;

(D32) $-\frac{E^{2}}{12}+\frac{4 E}{3}+\frac{4}{3 E}-\frac{1}{12 E^{2}}-\frac{5}{2}$

(C33) $E H[2,6]: \operatorname{RATEXPAND}(E H[2,4]+E H[2,2] * E H[4,2] / 90)$;

(D33) $\frac{E^{3}}{90}-\frac{3 E^{2}}{20}+\frac{3 E}{2}+\frac{3}{2 E}-\frac{3}{20 E^{2}}+\frac{1}{90 E^{3}}-\frac{49}{18}$

(C34) $\mathrm{F} 6(E H[2,6], 8,3,2)$;

$$
\operatorname{RATEXPAND}\left(56 X^{6}-\frac{56 H^{2} X^{6}+72 H^{8}}{H^{2}}\right)=-72 H^{6}
$$

(C35) DISPFUN(F6, F3, F2, F4);

(E35) $\mathrm{F} 6(G, J, N, M):=\operatorname{BLOCK}\left([], \mathrm{F} 3\left(\operatorname{RATEXPAND}\left(G X^{J}\right), N\right)\right.$, 
(E36) $\mathrm{F} 3(G, N):=\operatorname{BLOCK}([], \mathrm{A}: \operatorname{PART}(G, 2 N+1)$,

For $J$ through $N$ do $(\mathrm{Al}: \operatorname{PART}(G, J), \mathrm{A} 2: \operatorname{PART}(G, 2 N+1-J)$,

A3: NUMFACTOR(A1), A4: NUMFACTOR(A2), A1: $\frac{\mathrm{A} 1}{\mathrm{~A} 3}, \mathrm{~A} 2: \frac{\mathrm{A} 2}{\mathrm{A4}}$,

if $J \# N$ then A: A $+\mathrm{A} 3 \mathrm{~F} 2(\mathrm{~A} 1)+\mathrm{A} 4 \mathrm{~F} 4(\mathrm{~A} 2) \mathrm{ELSE} \mathrm{A}: \mathrm{A}+\mathrm{A} 3(X+$ $\left.H)^{\text {PART(A 1,2,2) }}+\mathrm{A} 4(X-H)^{\text {PART(A 2,1,2) }}\right)$, A: $\left.\operatorname{RATSIMP}(A)\right)$

(E37) $\quad \mathrm{F} 2(G):=(\operatorname{PART}(G, 2,1)+H \operatorname{PART}(G, 1,2))^{\operatorname{PART}(G, 2,2)}$

(E38) $\quad \mathrm{F} 4(G):=(\operatorname{PART}(G, 1,1)-H \operatorname{PART}(G, 2,2))^{\operatorname{PART}(G, 1,2)}$

TABLE 1

Weights at $t_{j}$ for second order approximations to derivatives of orders $p=1$ to 10 at $t=t_{0}=0$

\begin{tabular}{|c|c|c|c|c|c|c|}
\hline$p$ & 0 & 1 & 2 & 3 & 4 & 5 \\
\hline 1 & 0 & 1 & 0 & 0 & 0 & 0 \\
\hline 2 & -2 & 1 & 0 & 0 & 0 & 0 \\
\hline 3 & 0 & -1 & $\frac{1}{2}$ & 0 & 0 & 0 \\
\hline 4 & 6 & -4 & 1 & 0 & 0 & 0 \\
\hline 5 & 0 & $\frac{5}{2}$ & -2 & $\frac{1}{2}$ & 0 & 0 \\
\hline 6 & -20 & 15 & -6 & 1 & 0 & 0 \\
\hline 7 & 0 & -7 & 7 & -3 & $\frac{1}{2}$ & 0 \\
\hline 8 & 70 & -56 & 28 & -8 & 1 & 0 \\
\hline 9 & 0 & 21 & -24 & $\frac{27}{2}$ & -4 & $\frac{1}{2}$ \\
\hline 10 & -252 & 210 & -120 & 45 & -10 & 1 \\
\hline
\end{tabular}


TABLE 2

Weights at $t_{j}$ for fourth order approximations to derivatives of orders $p=1$ to 10 at $t=t_{0}=0$

\begin{tabular}{l|ccccccc}
$p$ & 0 & 1 & 2 & 3 & 4 & 5 & 6 \\
\hline 1 & 0 & $\frac{2}{3}$ & $-\frac{1}{12}$ & 0 & 0 & 0 & 0 \\
2 & $-\frac{5}{2}$ & $\frac{4}{3}$ & $-\frac{1}{12}$ & 0 & 0 & 0 & 0 \\
3 & 0 & $-\frac{13}{8}$ & 1 & $-\frac{1}{8}$ & 0 & 0 & 0 \\
4 & $\frac{28}{3}$ & $-\frac{13}{2}$ & 2 & $-\frac{1}{6}$ & 0 & 0 & 0 \\
5 & 0 & $\frac{29}{6}$ & $-\frac{13}{3}$ & $\frac{3}{2}$ & $-\frac{1}{6}$ & 0 & 0 \\
6 & $-\frac{75}{2}$ & 29 & -13 & 3 & $-\frac{1}{4}$ & 0 & 0 \\
7 & 0 & $-\frac{63}{4}$ & 17 & $-\frac{69}{8}$ & $\frac{13}{6}$ & $-\frac{5}{24}$ & 0 \\
8 & 154 & -126 & 68 & -23 & $\frac{13}{3}$ & $-\frac{1}{3}$ & 0 \\
9 & 0 & 54 & $-\frac{261}{4}$ & 41 & -15 & 3 & $-\frac{1}{4}$ \\
10 & -637 & 540 & $-\frac{1305}{4}$ & $\frac{410}{3}$ & $-\frac{75}{2}$ & 6 & $-\frac{5}{12}$
\end{tabular}

TABLE 3

Weights at $t_{j}$ for sixth order approximations to derivatives of orders $p=1$ to 10 at $t=t_{0}=0$

\begin{tabular}{c|cccccccc}
\hline & 0 & 1 & 2 & 3 & 4 & 5 & 6 & 7 \\
\hline 1 & 0 & $\frac{3}{4}$ & $-\frac{3}{20}$ & $\frac{1}{60}$ & 0 & 0 & 0 & 0 \\
2 & $-\frac{49}{18}$ & $\frac{3}{2}$ & $-\frac{3}{20}$ & $\frac{1}{90}$ & 0 & 0 & 0 & 0 \\
3 & 0 & $-\frac{61}{30}$ & $\frac{169}{120}$ & $-\frac{3}{10}$ & $\frac{7}{240}$ & 0 & 0 & 0 \\
4 & $\frac{91}{8}$ & $-\frac{122}{15}$ & $\frac{169}{60}$ & $-\frac{2}{5}$ & $\frac{7}{240}$ & 0 & 0 & 0 \\
5 & 0 & $\frac{323}{48}$ & $-\frac{13}{2}$ & $\frac{87}{32}$ & $-\frac{19}{36}$ & $\frac{13}{288}$ & 0 & 0 \\
6 & $-\frac{1023}{20}$ & $\frac{323}{8}$ & $-\frac{39}{2}$ & $\frac{87}{16}$ & $-\frac{19}{24}$ & $\frac{13}{240}$ & 0 & 0 \\
7 & 0 & $-\frac{971}{40}$ & $\frac{885}{32}$ & $-\frac{755}{48}$ & $\frac{601}{120}$ & $-\frac{41}{48}$ & $\frac{31}{480}$ & 0 \\
8 & $\frac{7007}{30}$ & $-\frac{971}{5}$ & $\frac{885}{8}$ & $-\frac{755}{18}$ & $\frac{601}{60}$ & $-\frac{41}{30}$ & $\frac{31}{360}$ & 0 \\
9 & 0 & $\frac{7323}{80}$ & $-\frac{1153}{10}$ & $\frac{6283}{80}$ & $-\frac{166}{5}$ & $\frac{139}{16}$ & $-\frac{13}{10}$ & $\frac{7}{80}$ \\
10 & -1066 & $\frac{7323}{8}$ & $-\frac{1153}{2}$ & $\frac{6283}{24}$ & -83 & $\frac{139}{8}$ & $-\frac{13}{6}$ & $\frac{1}{8}$
\end{tabular}




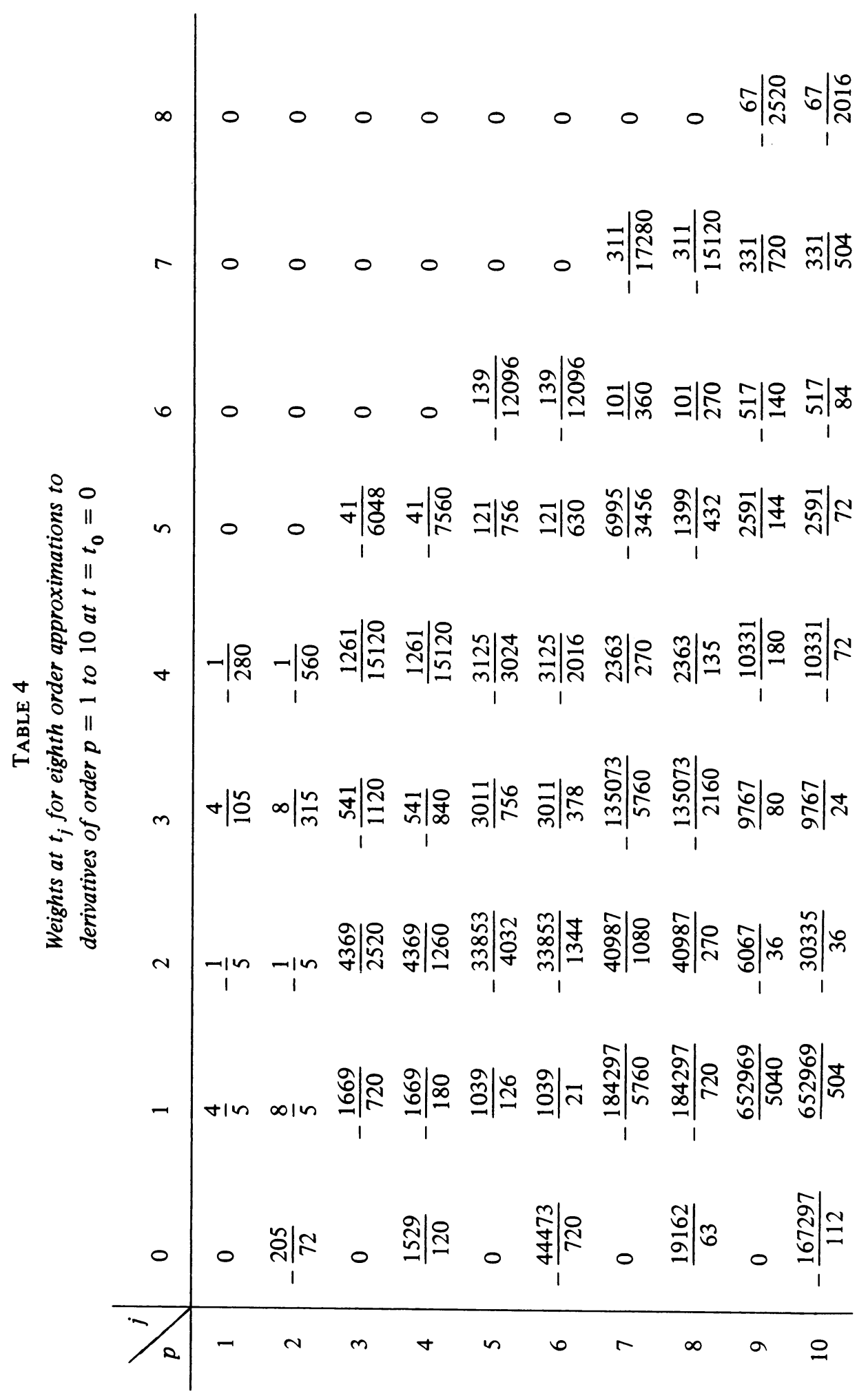




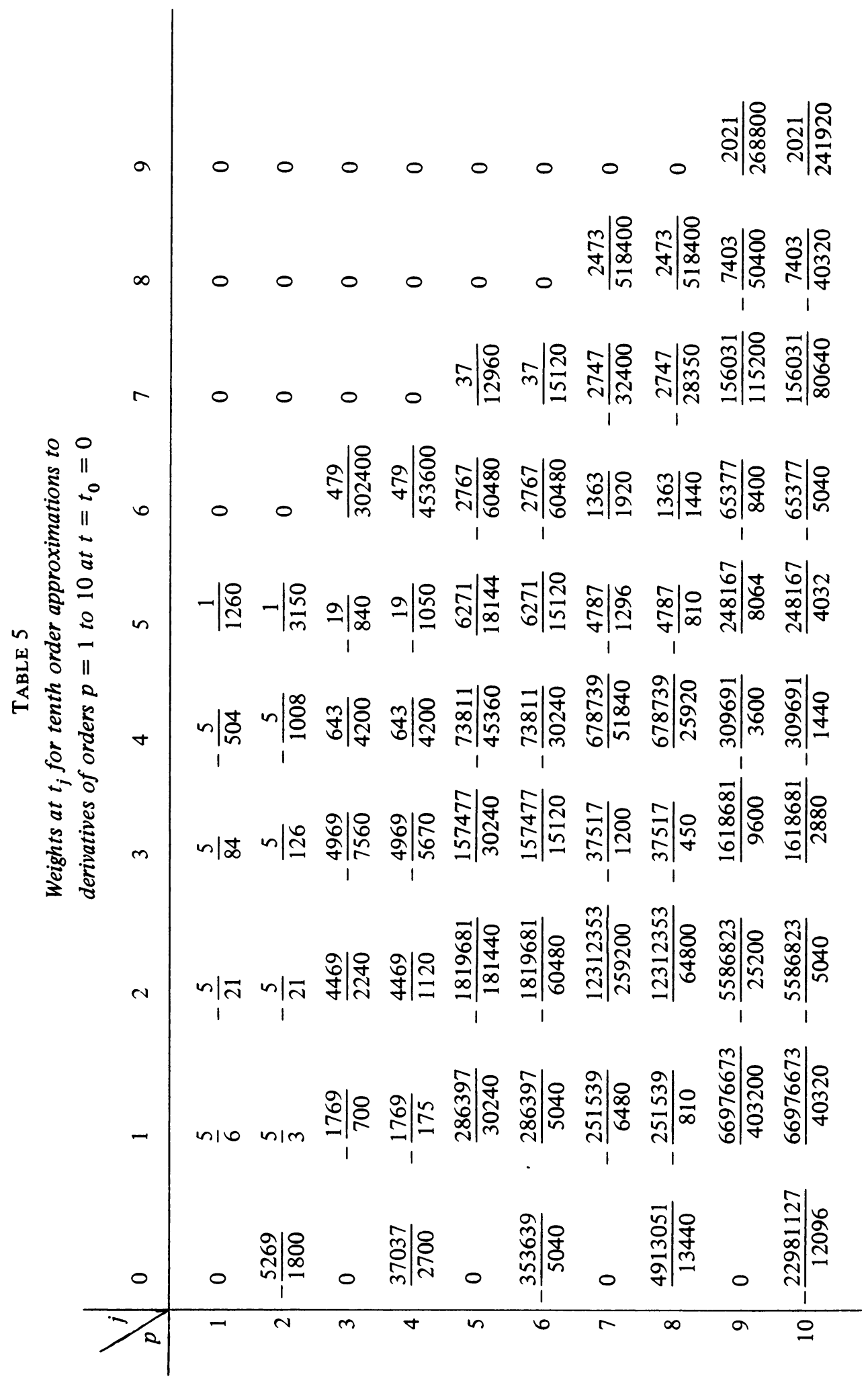


TABLE 6

Weights at $t_{j}$ for second order approximations to derivatives of orders $p=1$ to 10 at $t=t_{1 / 2}=h / 2$

\begin{tabular}{l|rrrrrr}
$p$ & 1 & 2 & 3 & 4 & 5 & 6 \\
\hline 1 & 1 & 0 & 0 & 0 & 0 & 0 \\
2 & $-\frac{1}{2}$ & $\frac{1}{2}$ & 0 & 0 & 0 & 0 \\
3 & -3 & 1 & 0 & 0 & 0 & 0 \\
4 & 1 & $-\frac{3}{2}$ & $\frac{1}{2}$ & 0 & 0 & 0 \\
5 & 10 & -5 & 1 & 0 & 0 & 0 \\
6 & $-\frac{5}{2}$ & $\frac{9}{2}$ & $-\frac{5}{2}$ & $\frac{1}{2}$ & 0 & 0 \\
7 & -35 & 21 & -7 & 1 & 0 & 0 \\
8 & 7 & -14 & 10 & $-\frac{7}{2}$ & $\frac{1}{2}$ & 0 \\
9 & 10 & -84 & 36 & -9 & 1 & 0 \\
10 & -21 & 45 & $-\frac{75}{2}$ & $\frac{35}{2}$ & $-\frac{9}{2}$ & $\frac{1}{2}$
\end{tabular}

TABLE 7

Weights at $t_{j}$ for fourth order approximations to derivatives of orders $p=1$ to 10 at $t=t_{1 / 2}=h / 2$

\begin{tabular}{l|ccccccc}
$p \backslash j$ & 1 & 2 & 3 & 4 & 5 & 6 & 7 \\
\hline 1 & $\frac{9}{8}$ & $-\frac{1}{24}$ & 0 & 0 & 0 & 0 & 0 \\
2 & $-\frac{17}{24}$ & $\frac{13}{16}$ & $-\frac{5}{48}$ & 0 & 0 & 0 & 0 \\
3 & $-\frac{17}{4}$ & $\frac{13}{8}$ & $-\frac{1}{8}$ & 0 & 0 & 0 & 0 \\
4 & $\frac{83}{48}$ & $-\frac{45}{16}$ & $\frac{59}{48}$ & $-\frac{7}{48}$ & 0 & 0 & 0 \\
5 & $\frac{415}{24}$ & $-\frac{75}{8}$ & $\frac{59}{24}$ & $-\frac{5}{24}$ & 0 & 0 & 0 \\
6 & $-\frac{41}{8}$ & $\frac{39}{4}$ & $-\frac{25}{4}$ & $\frac{29}{16}$ & $-\frac{3}{16}$ & 0 & 0 \\
7 & $-\frac{287}{4}$ & $\frac{91}{2}$ & $-\frac{35}{2}$ & $\frac{29}{8}$ & $-\frac{7}{24}$ & 0 & 0 \\
8 & $\frac{133}{8}$ & $-\frac{277}{8}$ & $\frac{435}{16}$ & $-\frac{553}{48}$ & $\frac{41}{16}$ & $-\frac{11}{48}$ & 0 \\
9 & $\frac{1197}{4}$ & $-\frac{831}{4}$ & $\frac{783}{8}$ & $-\frac{237}{8}$ & $\frac{41}{8}$ & $-\frac{3}{8}$ & 0 \\
10 & $-\frac{227}{4}$ & $\frac{2007}{16}$ & $-\frac{5375}{48}$ & $\frac{1421}{24}$ & $-\frac{153}{8}$ & $\frac{167}{48}$ & $-\frac{13}{48}$
\end{tabular}


TABLE 8

Weights at $t_{j}$ for sixth order approximations to

derivatives of orders $p=1$ to 10 at $t=t_{1 / 2}=h / 2$

\begin{tabular}{|c|c|c|c|c|c|c|c|c|}
\hline 1 & 1 & 2 & 3 & 4 & 5 & 6 & 7 & 8 \\
\hline 1 & $\frac{665}{576}$ & $-\frac{65}{1152}$ & $\frac{17}{5760}$ & 0 & 0 & 0 & 0 & 0 \\
\hline 2 & $-\frac{1891}{2304}$ & $\frac{1299}{1280}$ & $-\frac{499}{2304}$ & $\frac{259}{11520}$ & 0 & 0 & 0 & 0 \\
\hline 3 & $-\frac{1891}{384}$ & $\frac{1299}{640}$ & $-\frac{499}{1920}$ & $\frac{37}{1920}$ & 0 & 0 & 0 & 0 \\
\hline 4 & $\frac{4307}{1920}$ & $-\frac{1229}{320}$ & $\frac{377}{192}$ & $-\frac{1547}{3840}$ & $\frac{47}{1280}$ & 0 & 0 & 0 \\
\hline 5 & $\frac{4307}{192}$ & $-\frac{1229}{96}$ & $\frac{377}{96}$ & $-\frac{221}{384}$ & $\frac{47}{1152}$ & 0 & 0 & 0 \\
\hline 6 & $-\frac{4743}{640}$ & $\frac{1875}{128}$ & $-\frac{2645}{256}$ & $\frac{2855}{768}$ & $-\frac{867}{1280}$ & $\frac{209}{3840}$ & 0 & 0 \\
\hline 7 & $-\frac{33201}{320}$ & $\frac{4375}{64}$ & $-\frac{3703}{128}$ & $\frac{2855}{384}$ & $-\frac{2023}{1920}$ & $\frac{133}{1920}$ & 0 & 0 \\
\hline 8 & $\frac{25541}{960}$ & $-\frac{73063}{1280}$ & $\frac{110545}{2304}$ & $-\frac{133427}{5760}$ & $\frac{4253}{640}$ & $-\frac{12221}{11520}$ & $\frac{871}{11520}$ & 0 \\
\hline 9 & $\frac{76623}{160}$ & $-\frac{219189}{640}$ & $\frac{22109}{128}$ & $-\frac{19061}{320}$ & $\frac{4253}{320}$ & $-\frac{1111}{640}$ & $\frac{67}{640}$ & 0 \\
\hline 10 & $-\frac{25539}{256}$ & $\frac{173413}{768}$ & $-\frac{54359}{256}$ & $\frac{31507}{256}$ & $-\frac{11903}{256}$ & $\frac{2867}{256}$ & $-\frac{403}{256}$ & $\frac{77}{768}$ \\
\hline
\end{tabular}




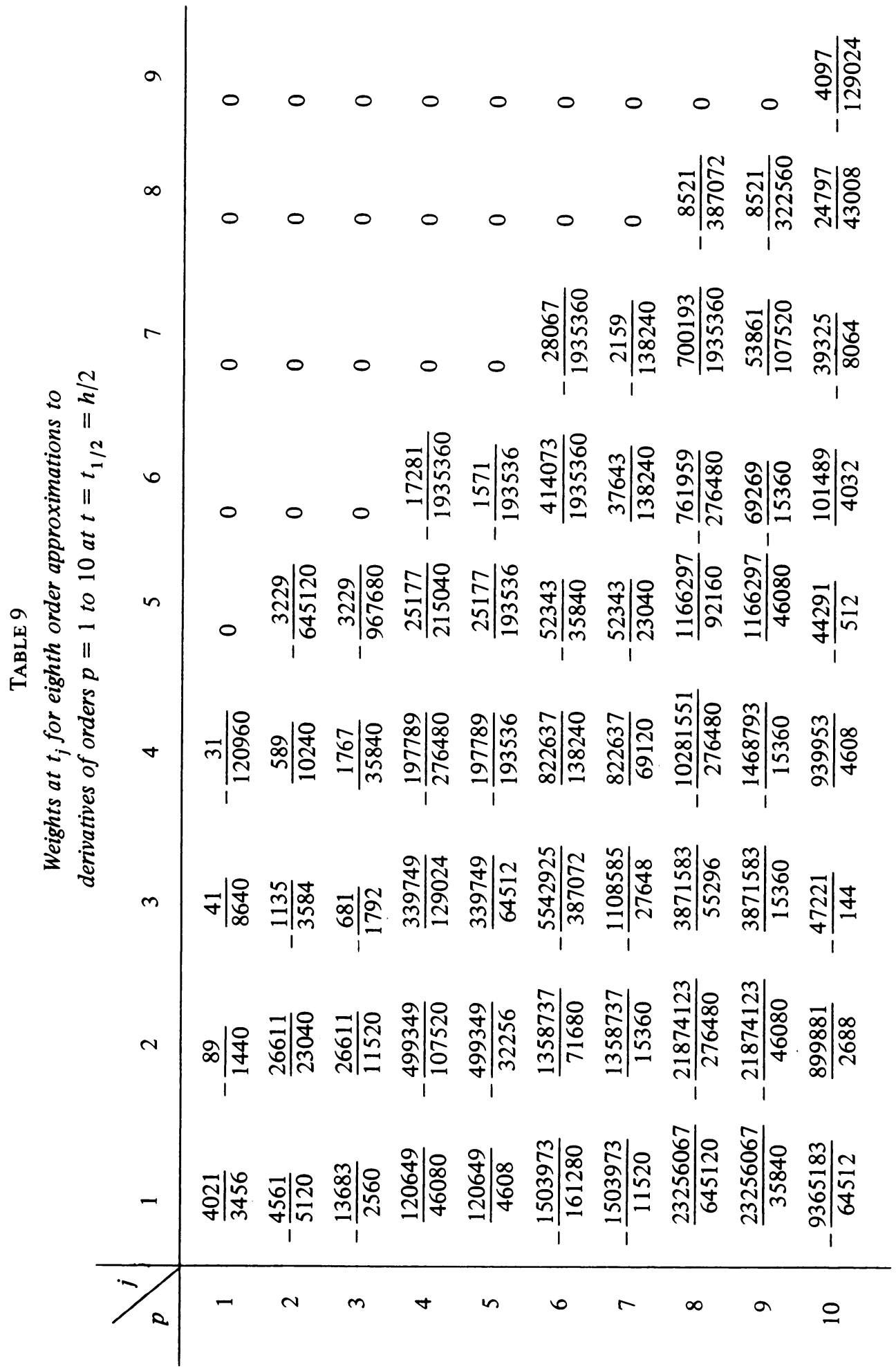




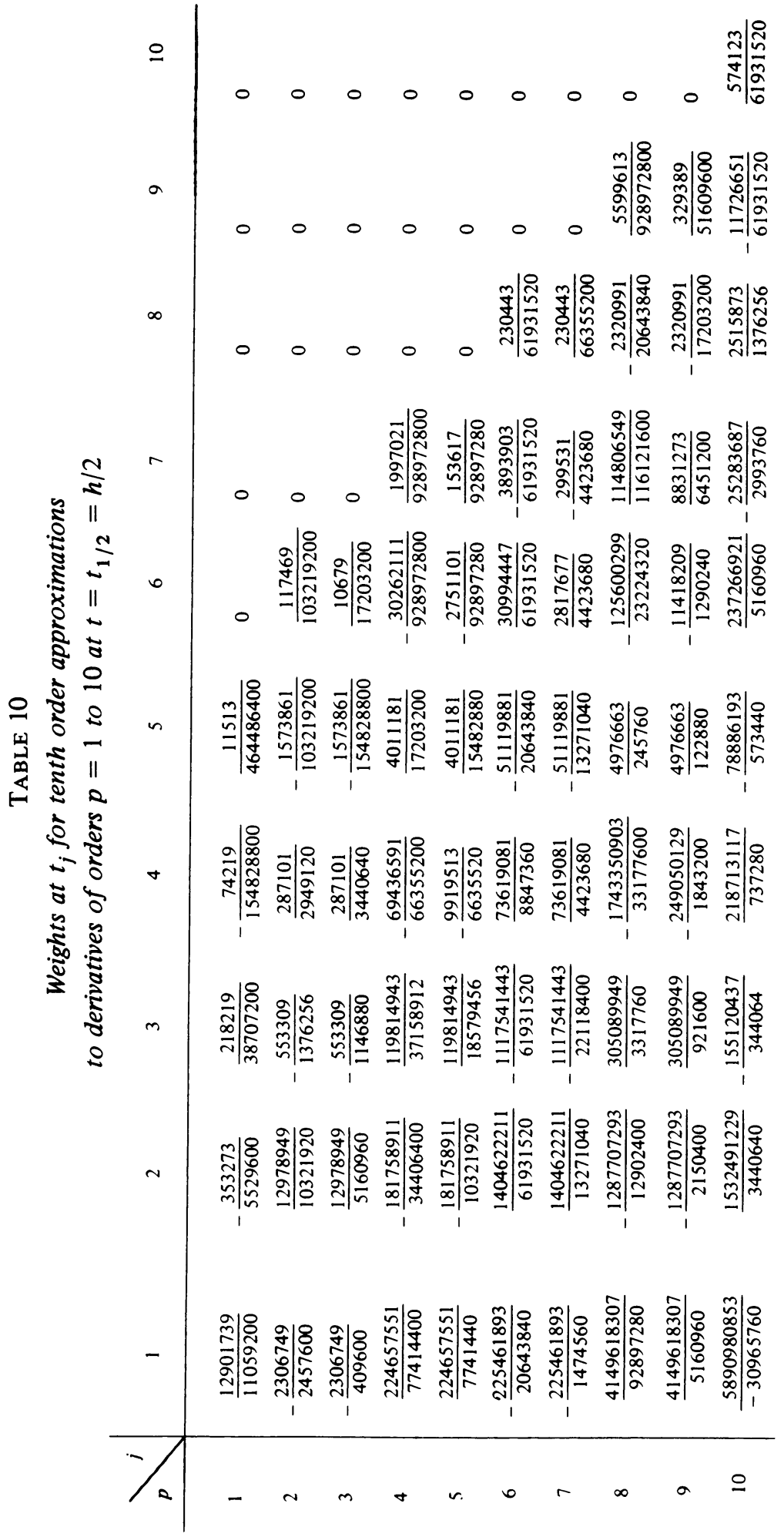


TABLE 11

Coefficients $C_{2 k, k}^{p, e}$

\begin{tabular}{c|cccc}
\hline$k$ & 1 & 2 & 3 & 4 \\
\hline 1 & $\frac{1}{6}$ & $-\frac{1}{30}$ & $\frac{1}{140}$ & $-\frac{1}{630}$ \\
2 & $\frac{1}{12}$ & $-\frac{1}{90}$ & $\frac{1}{560}$ & $-\frac{1}{3150}$ \\
3 & $\frac{1}{4}$ & $-\frac{7}{120}$ & $\frac{41}{3024}$ & $-\frac{479}{151200}$ \\
4 & $\frac{1}{6}$ & $-\frac{7}{240}$ & $\frac{41}{7560}$ & $-\frac{479}{453600}$ \\
5 & $\frac{1}{3}$ & $-\frac{13}{144}$ & $\frac{139}{6048}$ & $-\frac{37}{6480}$ \\
6 & $\frac{1}{4}$ & $-\frac{13}{240}$ & $\frac{139}{12096}$ & $-\frac{37}{15120}$ \\
7 & $\frac{5}{12}$ & $-\frac{31}{240}$ & $\frac{311}{8640}$ & $-\frac{2473}{259200}$ \\
8 & $\frac{1}{3}$ & $-\frac{31}{360}$ & $\frac{311}{15120}$ & $-\frac{2473}{518400}$ \\
9 & $\frac{1}{2}$ & $-\frac{7}{40}$ & $\frac{67}{1260}$ & $-\frac{2021}{134400}$ \\
10 & $\frac{5}{12}$ & $-\frac{1}{8}$ & $\frac{67}{2016}$ & $-\frac{2021}{241920}$ \\
& & & &
\end{tabular}

TABLE 12

Coefficients $C_{2 k, k}^{p, \phi}$

\begin{tabular}{c|cccc}
$p$ & 1 & 2 & 3 & 4 \\
\hline 1 & $\frac{1}{24}$ & $-\frac{17}{5760}$ & $\frac{31}{120960}$ & $-\frac{11513}{464486400}$ \\
2 & $\frac{5}{24}$ & $-\frac{259}{5760}$ & $\frac{3229}{322560}$ & $-\frac{117469}{51609600}$ \\
3 & $\frac{1}{8}$ & $-\frac{37}{1920}$ & $\frac{3229}{967680}$ & $-\frac{10679}{17203200}$ \\
4 & $\frac{7}{24}$ & $-\frac{47}{640}$ & $\frac{17281}{967680}$ & $-\frac{1997021}{464486400}$ \\
5 & $\frac{5}{24}$ & $-\frac{47}{1152}$ & $\frac{1571}{193536}$ & $-\frac{153617}{92897280}$ \\
6 & $\frac{3}{8}$ & $-\frac{209}{1920}$ & $\frac{28067}{967680}$ & $-\frac{230443}{30965760}$ \\
7 & $\frac{7}{24}$ & $-\frac{133}{1920}$ & $\frac{2159}{138240}$ & $-\frac{230443}{66355200}$ \\
8 & $\frac{11}{24}$ & $-\frac{871}{5760}$ & $\frac{8521}{193536}$ & $-\frac{5599613}{464486400}$ \\
9 & $\frac{3}{8}$ & $-\frac{67}{240}$ & $\frac{8521}{322560}$ & $-\frac{329389}{51609600}$
\end{tabular}


Department of Applied Mathematics

California Institute of Technology

Pasadena, California 91125

1. C. BALLESTER \& V. PEREYRA, "On the construction of discrete approximations to linear differential expressions," Math. Comp., v. 21, 1967, pp. 297-302.

2. A. BJÖRCK \& V. PEREYRA, "Solution of Vandermonde systems of equations," Math. Comp., v. 24, 1970, pp. 893-904.

3. E. ISAACSON \& H. B. KELLER, Analysis of Numerical Methods, Wiley, New York, 1966.

4. C. JORDAN, Calculus of Finite Differences, Chelsea, New York, 1947.

5. H. B. KELLER \& V. PEREYRA, "Difference methods and deferred corrections for ordinary boundary value problems," SIAM J. Numer. Anal. (To appear.)

6. MACSYMA, Reference Manual, The Mathlab Group, Project MAC, MIT, Boston, Mass., 1975.

7. M. ABRAMOWITZ \& I. A. STEGUN, Handbook of Mathematical Functions, National Bureau of Standards, Appl. Math. Series, no. 55, Washington, D. C., 1964.

8. W. G. BICKLEY, Math. Gaz., v. 25, 1941, pp. 19-27.

9. B. FORNBERG, "On a Fourier method for the integration of hyperbolic equations," SIAM J. Numer. Anal., v. 12, 1975, pp. 509-528. 